\title{
A Polarimetric Target Detector Using the Huynen Fork
}

\author{
Armando Marino, Shane R. Cloude, Fellow, IEEE, and Iain H. Woodhouse, Member, IEEE
}

\begin{abstract}
The contribution of synthetic aperture radar polarimetry in target detection is described and found to add valuable information. A new target detection methodology that makes novel use of the polarization fork of the target is described. The detector is based on a correlation procedure in the target space, and other target representations (e.g., Huynen parameters or $\alpha$ angle) can be employed. The mathematical formulation is general and can be applied to any kind of single target; however, in this paper, the detection is optimized for the odd and even bounces (the first two elements of the Pauli scattering vector) and for the oriented dipoles. Validation against real data shows significant agreement with the expected results based on the theoretical description.
\end{abstract}

Index Terms-Polarimetry, polarization fork (PF), synthetic aperture radar (SAR), target detection, target recognition.

\section{INTRODUCTION}

$\mathbf{T}$ HE ABILITY of synthetic aperture radar (SAR) to image through cloud cover and without solar illumination, in addition to its ability to partially penetrate foliage cover (dependent upon the wavelength), has established it as a powerful technique for target detection [1], [2]. In the last decade, attention has also been given to the examination of how the polarization of the signal may further develop this performance [3]-[7]. The aim of this paper is target detection exploiting a particular aspect of the polarimetric target response, namely, the polarization fork (PF), of the targets. The detector is not based on a statistical technique but rather on a physical approach based on the sensitivity of the polarimetric complex coherence to changes in polarization.

The approach is based on the potential to extract the target of interest in the target complex space. For this reason, full polarimetric data are required because they represent a basis for the target space [8], [9]. In some way, it acts similar to a decomposition theorem [10]; however, it is aimed more toward the detection of a chosen single target type rather than the breakdown of the partial target in predefined components.

The proposed algorithm is mainly focused on the detection of single (or simple) targets that can completely be characterized

Manuscript received June 1, 2009; revised September 30, 2009. First published February 17, 2010; current version published April 21, 2010. This work was conducted as a part of the SARTOM project led by eOsphere Limited and supported by the Electro-Magnetic Remote Sensing-Defence Technology Center (EMRS-DTC) under Contract EMRS/DTC/4/90.

A. Marino and I. H. Woodhouse are with The University of Edinburgh, EH9 3JZ Edinburgh, U.K. (e-mail: armando.marino@ed.ac.uk; i.h. woodhouse@ed.ac.uk).

S. R. Cloude is with AEL Consultants, EH12 9RG Edinburgh, U.K. (e-mail: aelc@mac.com).

Color versions of one or more of the figures in this paper are available online at http://ieeexplore.ieee.org.

Digital Object Identifier 10.1109/TGRS.2009.2038592 by one Sinclair (scattering) matrix [11]-[13]. In the case of a monostatic sensor and the reciprocity of the medium, the Sinclair matrix is symmetric and can be characterized by six parameters [13]-[15], e.g.,

$$
\begin{aligned}
{[S] } & =\left[R\left(\phi_{m}\right)\right]\left[T\left(\tau_{m}\right)\right]\left[S_{d}\right]\left[T\left(\tau_{m}\right)\right]\left[R\left(-\phi_{m}\right)\right] \\
{\left[S_{d}\right] } & =\left(\begin{array}{cc}
m e^{i(v+\zeta)} & 0 \\
0 & m \tan (\gamma) e^{-i(v-\zeta)}
\end{array}\right) \\
{\left[T\left(\tau_{m}\right)\right] } & =\left(\begin{array}{cc}
\cos \tau_{m} & -i \sin \tau_{m} \\
-i \sin \tau_{m} & \cos \tau_{m}
\end{array}\right) \\
{\left[R\left(\phi_{m}\right)\right] } & =\left(\begin{array}{cc}
\cos \phi_{m} & -\sin \phi_{m} \\
\sin \phi_{m} & \cos \phi_{m}
\end{array}\right) .
\end{aligned}
$$

In the work of Huynen [13], these parameters are linked to phenomenological aspects of the target. $\phi_{m}$ and $\tau_{m}$ are the orientation angle and the ellipticity angle of the target, and $m, v, \gamma$, and $\zeta$ are, respectively, the target magnitude, the target skip angle, the characteristic angle, and the absolute phase. Only five of them are sufficient to characterize a target since the target absolute phase $\varsigma$ can be neglected in singlepass polarimetry (note that $\varsigma$ is not negligible in polarimetric interferometry).

These parameters are related to the characteristic polarizations in the projective space of the Poincare sphere and can be represented by the PF [11], [16], [17]. The PF mainly consists of X-pol Nulls, Co-pol Nulls, and X-pol Max. The X-pol Nulls are polarizations that when transmitted do not have any return in the cross polarization (optimum polarizations). On the other hand, the Co-pol Nulls, when transmitted, do not have any return in the copolarization. Finally, the X-pol Max, when transmitted, has maximum cross-polarization return. The X-pol Nulls, Co-pol Nulls, and X-pol Max can always be visualized on the Poincaré sphere on the same plane (they form a fork shape). The reason why the PF is utilized is because it can represent physical target characteristics based on the location of the nulls. Fig. 1 represents the PF illustrating the link with four Huynen parameters (absolute magnitude and phase are not represented on the Poincaré sphere), where $X_{1}, X_{2}$ are X-pol Nulls, $C_{1}, C_{2}$ are Co-pol Nulls, and $S_{1}, S_{2}$ are X-pol Max.

The matrix representation (Sinclair matrix) can be modified as a vectorial [18], [19]

$$
\underline{k}=\frac{1}{2} \operatorname{Trace}([S] \Psi)=\left[k_{1}, k_{2}, k_{3}, k_{4}\right]^{T}
$$

where Trace(.) is the sum of the diagonal elements of the matrix inside, and $\Psi$ is a complete set of $2 \times 2$ basis matrices under a Hermitian inner product. In the case of reciprocal 


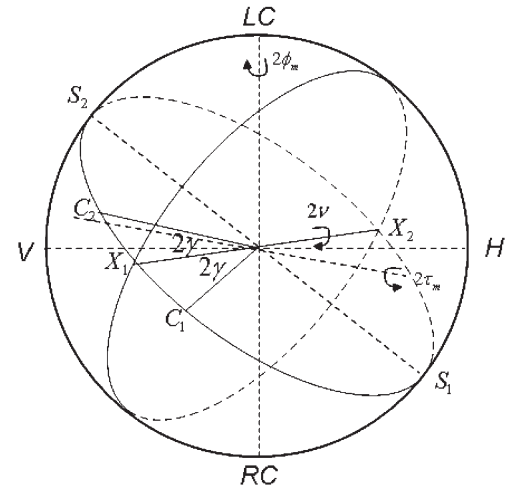

Fig. 1. PF and relationship with Huynen parameters.

medium and monostatic sensor, $\underline{k}$ is 3 -D complex $(S U(3))$ [20]. Finally, it is possible to define the scattering mechanism (weight vector) as a normalized vector $\underline{\omega}=\underline{k} /|\underline{k}|$. It is always possible to construct $\underline{\omega}$ starting from its PF.

In addition to the PF and Huynen parameters, other kinds of parameterizations are possible as long as the scattering mechanism can be reconstructed. In this context, a largely used procedure employs the $\alpha$ angle [21] as

$$
\underline{\omega}=\left[\cos \alpha, \sin \alpha \cos \beta e^{i \varepsilon}, \sin \alpha \sin \beta e^{i \mu}\right]^{T}
$$

where $\alpha$ is a characteristic angle (different from $\gamma$ ), and $\beta$ is dependent on the orientation of the target about the radar line of sight [21].

The target observed by a SAR system is not an idealized scattering target but a combination of different targets that we refer to as a partial target [22]-[24]. Decomposition theorems are able to represent the partial target as a combination of idealized single-target components [10]. To characterize a partial target, the single-scattering matrix is not sufficient since the partial target is a stochastic process, and second-order statistics are required. In this context, the target coherency matrix can be estimated as

$$
[C]=\left\langle\underline{k} \cdot \underline{k}^{* T}\right\rangle
$$

where $\langle$.$\rangle is the finite averaging operator. (Note that we are$ not employing interferometry but rather a single flight pass.) A classical formulation is when $k$ is expressed in Pauli basis (i.e., $\underline{k}_{P}=1 / \sqrt{2}\left[S_{H H}+S_{V V}, S_{H H}-S_{V V}, 2 S_{H V}\right]^{T}$, where $H$ is for horizontal, and $V$ is for vertical) or in lexicographic basis (i.e., $\underline{k}_{L}=\left[S_{H H}, \sqrt{2} S_{H V}, S_{V V}\right]^{T}$ ). In general, if the scattering vector in a generic basis is $\underline{k}=\left[k_{1}, k_{2}, k_{3}\right]^{T}$, where $k_{1}, k_{2}$, and $k_{3}$ are complex numbers, then the coherency matrix is

$$
[C]=\left[\begin{array}{ccc}
\left\langle\left|k_{1}\right|^{2}\right\rangle & \left\langle k_{1} k_{2}^{*}\right\rangle & \left\langle k_{1} k_{3}^{*}\right\rangle \\
\left\langle k_{2} k_{1}^{*}\right\rangle & \left\langle\left|k_{2}\right|^{2}\right\rangle & \left\langle k_{2} k_{3}^{*}\right\rangle \\
\left\langle k_{3} k_{1}^{*}\right\rangle & \left\langle k_{3} k_{2}^{*}\right\rangle & \left\langle\left|k_{3}\right|^{2}\right\rangle
\end{array}\right] .
$$

The methodology of this paper takes advantage of the polarimetric coherence [25]. If two different scattering mechanisms $\underline{\omega}_{1}$ and $\underline{\omega}_{2}$ are considered, then the polarimetric coherence is

$$
\gamma=\frac{\left\langle i\left(\underline{\omega}_{1}\right) \cdot i^{*}\left(\underline{\omega}_{2}\right)\right\rangle}{\sqrt{\left\langle i\left(\underline{\omega}_{1}\right) \cdot i^{*}\left(\underline{\omega}_{1}\right)\right\rangle\left\langle i\left(\underline{\omega}_{2}\right) \cdot i^{*}\left(\underline{\omega}_{2}\right)\right\rangle}}
$$

where $i$ is the image evaluated as

$$
i\left(\underline{\omega}_{j}\right)=\underline{\omega}_{j}^{* T} \cdot \underline{k} \quad \text { with } j=1,2 .
$$

In terms of the target coherency matrix, the polarimetric coherence is

$$
\gamma=\frac{\underline{\omega}_{1}^{* T}\langle[C]\rangle \underline{\omega}_{2}}{\sqrt{\underline{\omega}_{1}^{* T}\langle[C]\rangle \underline{\omega}_{1} \cdot \underline{\omega}_{2}^{* T}\langle[C]\rangle \underline{\omega}_{2}}} .
$$

Please note that this coherence is only polarimetric and not interferometric.

\section{Methodology}

Any (normalized) single target can uniquely be represented in the target space by a scattering mechanism $\underline{\omega}$. The image obtained with (7) evaluates the scalar projection of the observed target $\underline{k}$ on the scattering mechanism to be detected $\underline{\omega}_{j}$ (e.g., sphere, dipole, etc.). When the two images $i\left(\underline{\omega}_{1}\right)$ and $i\left(\underline{\omega}_{2}\right)$ are similar, the amplitude of the polarimetric coherence $\gamma$ [see (6)] is high (by definition).

We want to demonstrate the following: Given a scattering mechanism $\underline{\omega}_{1}$ proportional to the target to be detected, and given a second scattering mechanism $\underline{\omega}_{2}$ close to $\underline{\omega}_{1}$ within the target space, the polarimetric coherence is high if in the averaging cell the component of interest (proportional to $\underline{\omega}_{1}$ ) is stronger than the other two orthogonal components.

1) To demonstrate the hypothesis, the first step is to define a basis for the target space where the target of interest is limited to just one component of the 3-D complex vector $\underline{k}$. Geometrically, this operation can be accomplished with a change of basis using a unitary matrix, which sets one axis exactly over the target of interest. In the following, the scattering mechanism after the change of basis is referred to as $\underline{\omega}_{T}=[1,0,0]^{T} . \underline{\omega}_{T}$ is the single target we want to detect (following the initial thesis $\underline{\omega}_{1}=\underline{\omega}_{T}$ ). The coherence matrix $[C]$ will be calculated starting from this new basis. The resulting image when the target $\underline{\omega}_{T}$ is selected is

$$
i\left(\underline{\omega}_{T}\right)=k_{1} .
$$

In (9), the other two components of the scattering vector (i.e., $k_{2}$ and $k_{3}$ ) are completely deleted. The projection of $\underline{k}$ over $\underline{\omega}_{T}$ selects the component of interest in the partial target; hence, the target to detect is just in the $k_{1}$ component. For this reason (in the new basis that sets $\underline{\omega}_{T}=[1,0,0]^{T}$ ), $k_{2}$ and $k_{3}$ are considered as clutter.

2) Second, the scattering mechanism $\underline{\omega}_{2}$ must be constructed close to $\underline{\omega}_{T}$. The latter is named the "pseudotarget" $\underline{\omega}_{P}$ (i.e., $\left.\underline{\omega}_{2}=\underline{\omega}_{P}\right) . \underline{\omega}_{P}$ is obtained by slightly moving the entire PF, since a slightly different PF characterizes a slightly different target. In the first attempt, the small rotation of the characteristic polarizations on the Poincaré sphere can be accomplished using the Huynen parameters. In other words, in (1), $\phi_{m}, \tau_{m}, v$, and $\gamma$ are substituted with $\phi_{m} \pm \Delta \phi_{m}$, $\tau_{m} \pm \Delta \tau_{m}, v \pm \Delta v$, and $\gamma \pm \Delta \gamma$, where $\Delta \phi_{m}, \Delta \tau_{m}, \Delta v$, and $\Delta \gamma$ are positive real numbers corresponding to a fraction (e.g., a 12th or a tenth) of the maximum value of 
the corespective variable. The variation can be positive or negative to keep the final variable within the allowed range of values. For the pseudotarget, (1) becomes

$$
\begin{aligned}
{\left[S_{P}\right]=} & {\left[R\left(\phi_{m}+\Delta \phi_{m}\right)\right]\left[T\left(\tau_{m}+\Delta \tau_{m}\right)\right]\left[S_{d}\right] } \\
& \times\left[T\left(\tau_{m}+\Delta \tau_{m}\right)\right]\left[R\left(-\left(\phi_{m}+\Delta \phi_{m}\right)\right]\right. \\
{\left[S_{d}\right]=} & \left(\begin{array}{cc}
e^{i(v+\Delta v)} & 0 \\
0 & \tan (\gamma+\Delta \gamma) e^{-i(v+\Delta v)}
\end{array}\right) .
\end{aligned}
$$

Consequently, $\left[S_{T}\right] \approx\left[S_{P}\right]$, where $\left[S_{T}\right]$ is the scattering matrix of the target to detect (i.e., (1) with $m=1$ and $\varsigma=$ $0)$. Similarly, $\underline{\omega}_{P}$ can be obtained starting from the $\alpha$ angle parameterization as

$$
\begin{aligned}
& \underline{\omega}_{T}=\left[\cos \alpha, \sin \alpha \cos \beta e^{i \varepsilon}, \sin \alpha \sin \beta e^{i \mu}\right]^{T} \\
& \underline{\omega}_{P}=\left[\begin{array}{c}
\cos (\alpha+\Delta \alpha) \\
\sin (\alpha+\Delta \alpha) \cos (\beta+\Delta \beta) e^{i(\varepsilon+\Delta \varepsilon)} \\
\sin (\alpha+\Delta \alpha) \sin (\beta+\Delta \beta) e^{i(\mu+\Delta \mu)}
\end{array}\right]
\end{aligned}
$$

where again $\Delta \alpha, \Delta \beta, \Delta \varepsilon$, and $\Delta \mu$ are a fraction of the maximum value of corespective variables (in case of $\varepsilon$ and $\mu$, the maximum value is fixed to $2 \pi$ ). Again, $\underline{\omega}_{T} \approx \underline{\omega}_{P}$.

In both parameterizations, the components of the scattering mechanism are not linearly linked with the parameters. However, fortunately, they are continuous functions; when the parameters are selected in the allowed range of values (i.e., products of continuous functions). This guarantees that if the parameter variation is small enough, then the change in the pseudotarget will be small as well. The optimization of the variations to have valuable pseudotarget components is studied in the following sections. Once the expression of $\omega_{P}$ in the basis used by the parameterization (e.g., Pauli for $\alpha$ ) is obtained, the same change of basis that makes $\underline{\omega}_{T}=[1,0,0]^{T}$ is performed over $\underline{\omega}_{P}$. Consequently, $\underline{\omega}_{P}=$ $[a, b, c]^{T}$, with $a, b$, and $c$ as complex numbers. Considering $\underline{\omega}_{T} \approx \underline{\omega}_{P}$, we have $|a| \approx 1,|b| \approx 0$, and $|c| \approx 0$.

To show the relationship with the Huynen parameters, the scattering mechanism for the target of interest can be calculated as

$$
\begin{aligned}
\underline{\omega}_{T} & =\frac{1}{2}[U(\varphi, \vartheta, \delta)] \operatorname{Trace}\left(\left[S\left(\phi_{m}, \tau_{m}, \gamma, v\right)\right] \Psi\right) \\
& =[1,0,0]^{T}
\end{aligned}
$$

where $[S]$ is calculated from (1) (the brackets show the dependence to the Huynen parameters). The $[U]$ matrix performs the change of basis that makes $\underline{\omega}_{T}=[1,0,0]^{T}$. $[U]$ depends on two rotation angles and a change of phase (i.e., $\varphi, \vartheta$, and $\delta$ ). The pseudotarget can be calculated with a slight change of the Huynen parameters as

$$
\begin{aligned}
\underline{\omega}_{P}= & \frac{1}{2}[U(\varphi, \vartheta, \delta)] \\
& \cdot \operatorname{Trace}\left(\left[S \left(\phi_{m}+\Delta \phi_{m}, \tau_{m}\right.\right.\right. \\
& \left.\left.\left.\quad+\Delta \tau_{m}, \gamma+\Delta \gamma, v+\Delta v\right)\right] \Psi\right) \\
= & {[a, b, c]^{T} . }
\end{aligned}
$$

Hence, if $\Delta \phi_{m}=\Delta \tau_{m}=\Delta v=\Delta \gamma=0$, then $\underline{\omega}_{T}=\underline{\omega}_{P}$; on the other hand, if the variations are small, then the two scattering mechanisms start to be different, introducing the required distance.

3) Once the two scattering mechanisms are defined, the polarimetric coherence (in the new basis) can be estimated from

$$
\gamma\left(\underline{\omega}_{T}, \underline{\omega}_{P}\right)=\frac{\left\langle i\left(\underline{\omega}_{T}\right) \cdot i^{*}\left(\underline{\omega}_{P}\right)\right\rangle}{\sqrt{\left\langle i\left(\underline{\omega}_{T}\right) \cdot i^{*}\left(\underline{\omega}_{T}\right)\right\rangle\left\langle i\left(\underline{\omega}_{P}\right) \cdot i^{*}\left(\underline{\omega}_{P}\right)\right\rangle}}
$$

where

$$
\begin{aligned}
\left\langle i\left(\underline{\omega}_{T}\right) \cdot i^{*}\left(\underline{\omega}_{P}\right)\right\rangle= & a\left\langle\left|k_{1}\right|^{2}\right\rangle+b\left\langle k_{1} \cdot k_{2}^{*}\right\rangle+c\left\langle k_{1} \cdot k_{3}^{*}\right\rangle \\
\left\langle i\left(\underline{\omega}_{T}\right) \cdot i^{*}\left(\underline{\omega}_{T}\right)\right\rangle= & \left\langle\left|k_{1}\right|^{2}\right\rangle \\
\left\langle i\left(\underline{\omega}_{P}\right) \cdot i_{*}\left(\underline{\omega}_{P}\right)\right\rangle= & |a|^{2}\left\langle\left|k_{1}\right|^{2}\right\rangle+|b|^{2}\left\langle\left|k_{2}\right|^{2}\right\rangle+|c|^{2}\left\langle\left|k_{3}\right|^{2}\right\rangle \\
& +2 \operatorname{Re}\left(a b^{*}\left\langle k_{1} \cdot k_{2}^{*}\right\rangle\right)+2 \operatorname{Re}\left(a c^{*}\left\langle k_{1} \cdot k_{3}^{*}\right\rangle\right) \\
& +2 \operatorname{Re}\left(c b^{*}\left\langle k_{3} \cdot k_{2}^{*}\right\rangle\right) .
\end{aligned}
$$

After dividing both numerator and denominator by $|a|\left\langle\left|k_{1}\right|^{2}\right\rangle$, the amplitude of the polarimetric coherence becomes (16) and (17), shown at the bottom of the page. We refer to $|b| /|a|$ and $|c| /|a|$ as reduction ratios $(\operatorname{Re} d R)$. The pseudotargets are chosen to have small RedR. Hence, in the sum, the elements containing $R e d R$ are lowered. These terms are referred to as clutter terms. In (16) and (17), they are all of the elements except the ones with the sought component $k_{1}$ alone (i.e., $\left\langle\left|k_{1}\right|^{2}\right\rangle$ that after the division becomes 1). There exist two typologies of clutter terms: cross correlations and powers. The cross correlations are generally small, since for partial targets the components of $\underline{k}$ are likely to be uncorrelated. The power terms depend on the power of the clutter $k_{2}$ and $k_{3}$. Finally, when $\left\langle\left|k_{1}\right|^{2}\right\rangle$ is

$$
\begin{aligned}
\left|\gamma\left(\underline{\omega}_{T}, \underline{\omega}_{P}\right)\right| & =\frac{\left|1+\frac{b}{|a|} \frac{\left\langle k_{1} \cdot k_{2}^{*}\right\rangle}{\left\langle\left|k_{1}\right|^{2}\right\rangle}+\frac{c}{|a|} \frac{\left\langle k_{1} \cdot k_{3}^{*}\right\rangle}{\left\langle\left|k_{1}\right|^{2}\right\rangle}\right|}{\sqrt{\Lambda}} \\
\Lambda & =1+\frac{|b|^{2}}{|a|^{2}} \frac{\left\langle\left|k_{2}\right|^{2}\right\rangle}{\left\langle\left|k_{1}\right|^{2}\right\rangle}+\frac{|c|^{2}}{|a|^{2}} \frac{\left\langle\left|k_{3}\right|^{2}\right\rangle}{\left\langle\left|k_{1}\right|^{2}\right\rangle}+\frac{2 \operatorname{Re}\left(a b^{*}\left\langle k_{1} \cdot k_{2}^{*}\right\rangle\right)+2 \operatorname{Re}\left(a c^{*}\left\langle k_{1} \cdot k_{3}^{*}\right\rangle\right)+2 \operatorname{Re}\left(c b^{*}\left\langle k_{3} \cdot k_{2}^{*}\right\rangle\right)}{|a|^{2}\left\langle\left|k_{1}\right|^{2}\right\rangle}
\end{aligned}
$$




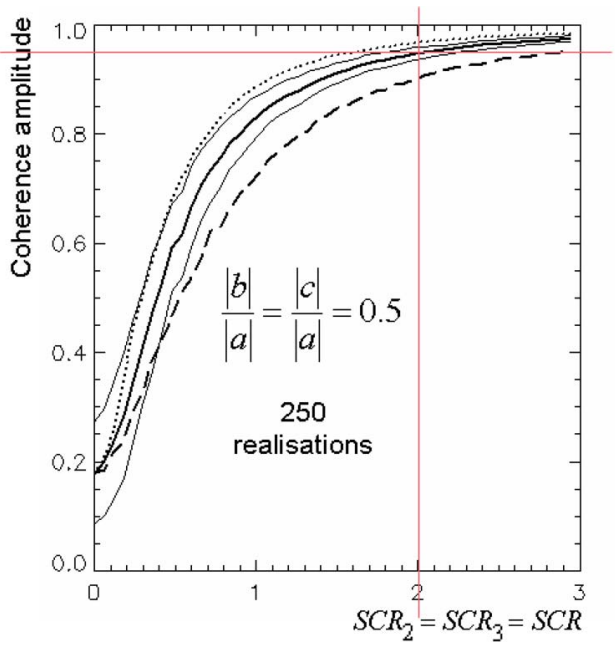

Fig. 2. Coherence amplitude detector. (Solid lines) Inside the standard deviation boundaries for uncorrelated target clutter. (Dotted line) Positive target-clutter correlation. (Dashed line) Negative target-clutter correlation. Average over 250 realizations with window $5 \times 5$.

higher than the clutter terms, the RedR combined with the division for $\left\langle\left|k_{1}\right|^{2}\right\rangle$ makes the clutter terms negligible in the sum, and the polarimetric coherence has unitary amplitude. If the component of interest is not dominant, then the clutter terms start to have more influence in the final sum, lowering the coherence amplitude.

4) In conclusion, the detector is obtained by setting a threshold on the coherence amplitude as

$$
\left|\gamma\left(\underline{\omega}_{T}, \underline{\omega}_{P}\right)\right|<T \text {. }
$$

Fig. 2 presents the simulation of the coherence amplitude estimated as a stochastic process consisting of a deterministic target $k_{1}$ (target to be detected) and two random variables, complex Gaussian zero mean (i.e., $k_{2}$ and $k_{3}$ ), independent of each other. The solid lines show the mean value of the coherence (over 250 realizations) confined in the standard deviation boundaries. A $5 \times 5$ window and a $R e d R$ of 0.5 are considered. The signal-to-clutter ratio $(S C R)$ is defined as $S C R_{j}=\left\langle\left|k_{1}\right|^{2}\right\rangle /\left\langle\left|k_{j}\right|^{2}\right\rangle$ with $j=2,3$. In the plot, both $S C R$ s are simultaneously increased, i.e., $S C R=S C R_{2}=$ $\mathrm{SCR}_{3}$.

\section{A. Bias Removal}

The solid line in Fig. 2 is obtained by considering the three components of the scattering vector $\underline{k}$ independently of each other; hence, the cross-correlation terms are almost zero (it is different from 0 just because the number of samples is finite). This condition is a good approximation for partial targets; however, it could not be fulfilled for single (coherent) targets. The dotted and dashed lines in Fig. 2 present the case when the coherent target is correlated with the two clutter components in constructive or destructive ways, respectively. The amplitude of the correlation coefficient between the target and the clutter is 0.65 . In conclusion, the correlation between target and clutter creates bias on the coherence amplitude. The aim of this section is to remove this bias. First, we recognize that the cross terms

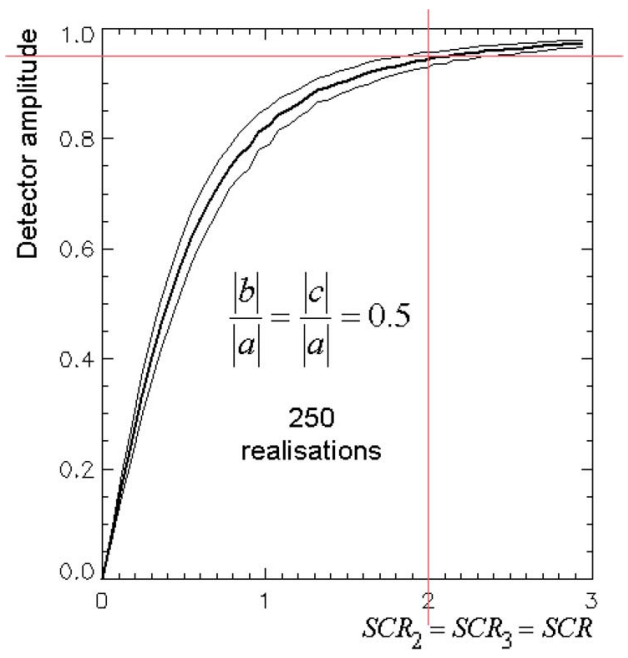

Fig. 3. Detector: mean over 250 realizations inside the standard deviation boundaries. Window $5 \times 5$.

do not add useful information for the proposed detector. In the case of uncorrelated $\underline{k}$ components, they merely add noise related to the finite averaging [25]. However, for high values of coherence, the bias introduced is not appreciable. On the other hand, when the $\underline{k}$ components are correlated, they bring bias that results in false alarms or misdetections. Consequently, the detector is improved and simplified when they are ignored.

To neglect them, the polarimetric coherence operator is substituted with another operator that works on the space of the target component power as

$$
\left|\gamma_{d}\left(\underline{\omega}_{T}, \underline{\omega}_{P}\right)\right|=\frac{\left|\underline{\omega}_{T}^{* T}\langle[P]\rangle \underline{\omega}_{P}\right|}{\sqrt{\underline{\omega}_{T}^{* T}\langle[P]\rangle \underline{\omega}_{T} \cdot \underline{\omega}_{P}^{* T}\langle[P]\rangle \underline{\omega}_{P}}}
$$

where

$$
\begin{aligned}
& {[P]=\left[\begin{array}{ccc}
\left\langle\left|k_{1}\right|^{2}\right\rangle & 0 & 0 \\
0 & \left\langle\left|k_{2}\right|^{2}\right\rangle & 0 \\
0 & 0 & \left\langle\left|k_{3}\right|^{2}\right\rangle
\end{array}\right]} \\
& \left|\gamma_{d}\left(\underline{\omega}_{T}, \underline{\omega}_{P}\right)\right|=\frac{1}{\sqrt{1+\frac{|b|^{2}}{|a|^{2}} \frac{\left\langle\left|k_{2}\right|^{2}\right\rangle}{\left\langle\left|k_{1}\right|^{2}\right\rangle}+\frac{|c|^{2}}{|a|^{2}} \frac{\left\langle\left|k_{3}\right|^{2}\right\rangle}{\left\langle\left|k_{1}\right|^{2}\right\rangle}}} .
\end{aligned}
$$

The modified coherence in (21) will be referred to as the detector. The latter is dependent simply on the power components of the scattering vector $\underline{k}$.

Looking at (21), the lowering effect played by the RedR is clear. If the clutter power is lower than the target power, then the two terms on the denominator are negligible, and $\left|\gamma_{d}\right|=1$. The trend of the detector can be seen in Fig. 3. Comparing Figs. 2 and 3, the variance appears strongly reduced for low SCRs; moreover, the two means look very close for values higher than 0.6. The difference for lower values is related with the coherence bias due to finite averaging. The bias is brought by the cross terms. Consequently, neglecting them, the bias disappears (please note, for very high values of clutter, the detector becomes 0 ). For uncorrelated components, the cross terms only result in increasing variance. 


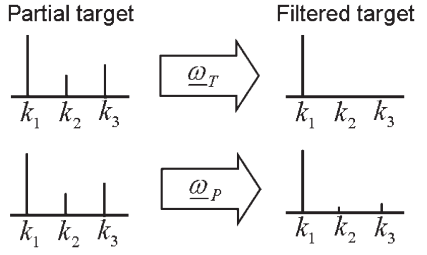

(a)

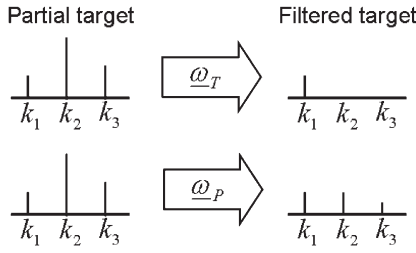

(b)
Fig. 4. Visual explanation of the filter with target and pseudotarget. (a) Detection achieved. (b) No detection achieved.

The final expression of the detector sets a threshold on (21) as

$$
\left|\gamma_{d}\left(\underline{\omega}_{T}, \underline{\omega}_{P}\right)\right|<T .
$$

\section{B. Detector Interpretation}

To give an intuitive view of the detector, Fig. 4 represents the filtering effect as a simple schematic. The vertical bars stand for the power of the scattering vector components. After the change of basis that makes $\underline{\omega}_{T}=[1,0,0]^{T}, k_{1}$ represents the target to detect, and $k_{2}, k_{3}$ are the clutter.

The final image (as interpreted by the final detector) is obtained as the incoherent sum of the three components. The image formation behaves similar to a filter (more precisely it is a scalar projection). The first row of any example (i.e., $\underline{\omega}_{T}$ filter) is ideal and completely deletes the orthogonal clutter components.

The second row (i.e., $\underline{\omega}_{P}$ filter) results in a linear combination of the sought component (slightly lowered) plus a small amount of the orthogonal ones. In (a), the match between the target and the pseudotarget final image is high since the power in the two images is similar. This is not true in (b), since the $\omega_{P}$ image has much more power than the $\omega_{T}$ image; hence, in the normalization in (21), the $\underline{\omega}_{P}$ image significantly lowers the coherence.

\section{Parameter Selection}

\section{A. Reduction Ratios (RedR) and Threshold}

The detector [as expressed in (21)] is a stochastic process [26]. To optimally set the threshold and assess the probability of false alarms and misdetection, the probability density function of the detector is required. Its evaluation is out of the scope of this first paper; hence, we are looking for an expression of the detector independent of the statistical realization. For this purpose, the finite average operator $\langle$.$\rangle is substituted with the$ expected value $E[$.$] . Considering that the detector works with a$ high value of coherence, the latter assumption is easily fulfilled for a $5 \times 5$ window as

$$
\begin{aligned}
\left|\gamma_{d}\left(\underline{\omega}_{T}, \underline{\omega}_{P}\right)\right| & \approx \frac{1}{\sqrt{1+\frac{|b|^{2}}{|a|^{2}} \frac{1}{S C R_{2}}+\frac{|c|^{2}}{|a|^{2}} \frac{1}{S C R_{3}}}} \\
S C R_{2} & =E\left[\left|k_{1}\right|^{2}\right] / E\left[\left|k_{2}\right|^{2}\right] \\
S C R_{3} & =E\left[\left|k_{1}\right|^{2}\right] / E\left[\left|k_{3}\right|^{2}\right] .
\end{aligned}
$$

Once the pseudotarget $\underline{\omega}_{P}$ is fixed, (23) is an expression exclusively related to the signal-to-clutter ratio $(S C R)$.

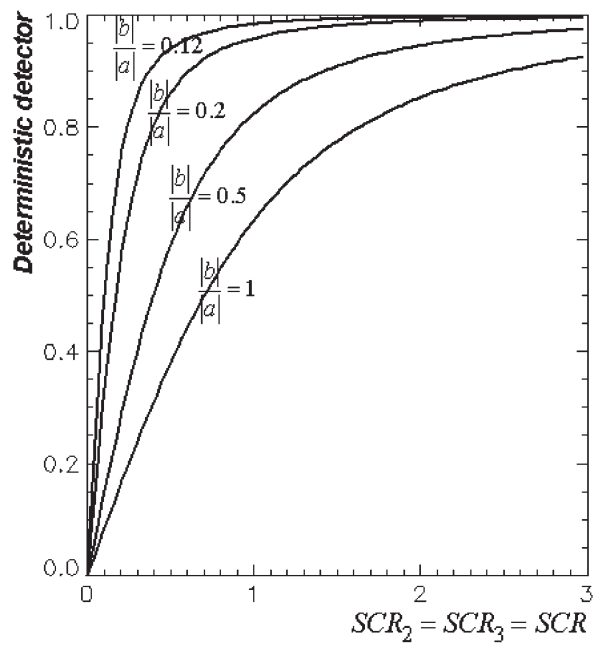

Fig. 5. Deterministic detector (reduction ratio varied).

Fig. 5 represents the results, where the value of the RedR is varied. Please note that the mean curves in Fig. 3 overlap almost perfectly with the one in Fig. 5 (for $\operatorname{Red} R=0.5$ ). The detector presents no bias, and the threshold can be set on the deterministic detector to the base of the $S C R$ to be detected. Fig. 5 also allowed some consideration of the RedR. The detector increases when the ratio is reduced (the clutter terms are lower). Regarding the choice of the ratio, a small value reduces the variance (since we work with higher values of modified coherence); however, the range of discrimination between targets is reduced (the curve flattens earlier). Considering we want to detect targets with $S C R$ higher than 1.5-2, a good choice for the ratio is 0.5 (which makes the threshold set at around 0.95).

Once the RedR is fixed, it is possible to set the threshold. For strong targets, the discrimination is quite easy, so the minimization of false alarm is the key point. Hence, a high $S C R$ can be chosen (this leads to a higher threshold). On the other hand, if embedded (e.g., foliage penetration FOLPEN) or weak targets (with low total backscattering) are to be detected, then a lower $S C R$ must be selected (consequently a lower threshold). The effect of the threshold choice is clearly visible in the validation section. Please note that the detection ability is not directly related to the total power scattered by the target (span of the scattering matrix) but exclusively with the reciprocal weight of the scattering components. The threshold reduction for weak targets is related to the noise effect, which confuses the polarimetric characteristics. To check this property, a simulation was performed with no clutter and just additive thermal uncorrelated noise. The result is that the threshold is required to be lower than 0.98 to detect a target of interest with SNR (over the window) of about $1 \mathrm{~dB}$ and less than 0.88 for $-10 \mathrm{~dB}$ SNR.

\section{B. Pseudotarget Selection}

In the previous section, a tacit hypothesis is employed: $|b|=$ $|c|$. The aim of this section is to evaluate the effects of $|b| \neq$ $|c|$. The components of $\underline{\omega}_{P}$ are not independent since $|a|^{2}+$ $|b|^{2}+|c|^{2}=1$, because $\underline{\omega}_{P}$ is a normalized vector. To show the 


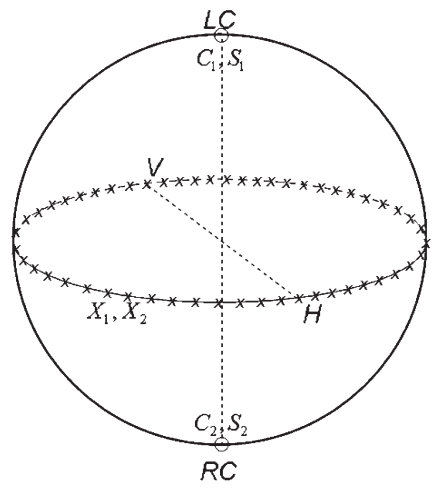

(a)

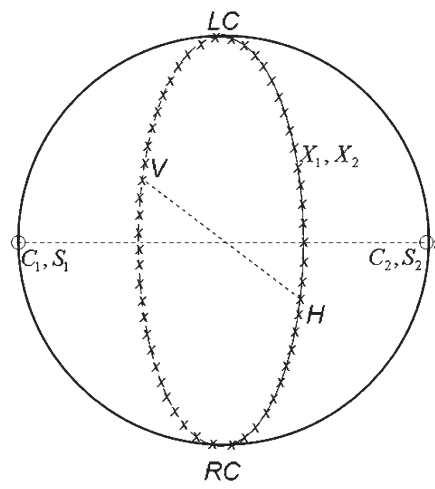

(b)

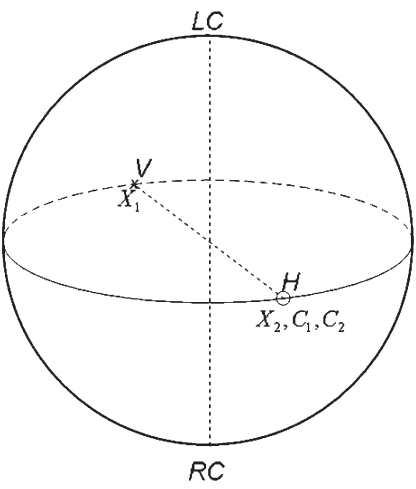

(c)

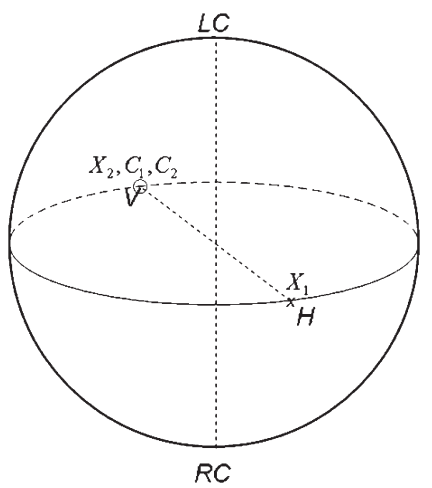

(d)

Fig. 6. Poincaré representation of single target detected. (a) Odd bounce. (b) Even bounce. (c) Vertical dipole. (d) Horizontal dipole.

importance of a good choice of $b$ and $c$, an example is presented. It is given that $\underline{k}=\kappa\left[a^{\prime}, b_{0}, 0\right], \underline{\omega}_{P}=\left[a, 0, c_{0}\right]$, and $\underline{\omega}_{T}=$ $[1,0,0]$, where $\left|b_{0}\right|=\sqrt{1-\left|a^{\prime}\right|^{2}}$, and $\left|c_{0}\right|=\sqrt{1-|a|^{2}}$. The detector will be

$$
\left|\gamma_{d}\left(\underline{\omega}_{T}, \underline{\omega}_{P}\right)\right|=\frac{1}{\sqrt{1+\frac{0}{|a|^{2}} \frac{\left|b_{0}\right|^{2}}{\left|a^{\prime}\right|^{2}}+\frac{\left|c_{0}\right|^{2}}{|a|^{2}} \frac{0}{\left|a^{\prime}\right|^{2}}}}=1 .
$$

Basically, the orthogonality (or in general the geometrical relationship) between the clutter components of $\underline{k}$ and $\underline{\omega}_{P}$ can bias the detector. To remove this bias, we want to find a relation between $b$ and $c$ that makes the detector not biased. It can be demonstrated that this choice is $|b|=|c|$. To show the feasibility, we consider a general target as $\underline{k}=\kappa\left[a^{\prime}, b^{\prime}, c^{\prime}\right]$. After algebraic manipulations, we have

$$
\left|\gamma_{d}\left(\underline{\omega}_{T}, \underline{\omega}_{P}\right)\right|=\frac{1}{\sqrt{1+\frac{|b|^{2}}{|a|^{2}\left|a^{\prime}\right|^{2}}\left(\left|b^{\prime}\right|^{2}+\left|c^{\prime}\right|^{2}\right)}} .
$$

Equation (26) states that the total (normalized) power of the clutter components is contained in $\left|b^{\prime}\right|^{2}+\left|c^{\prime}\right|^{2}$, and it does not matter which is the strongest component between $b^{\prime}$ and $c^{\prime}$, the bias is removed.

\section{Detector Implementation}

The expression obtained in (21) is still dependent on the basis used to express the vectors $\underline{\omega}_{T}$ and $\underline{\omega}_{P}$. In that basis, the target to detect is exclusively present in the $k_{1}$ component (i.e., $k_{2}, k_{3}$ represent the clutter). If three unitary vectors $\underline{e}_{1}=$ $[1,0,0]^{T}, \underline{e}_{2}=[0,1,0]^{T}$, and $\underline{e}_{3}=[0,0,1]^{T}$ are introduced, then the power of target and clutter (in the basis that makes $\left.\underline{\omega}_{T}=[1,0,0]^{T}\right)$ can be written as

$$
\begin{aligned}
P_{T} & =\left\langle\left|\underline{k}^{T} \cdot \underline{e}_{1}\right|^{2}\right\rangle \\
P_{C 2} & =\left\langle\left|\underline{k}^{T} \cdot \underline{e}_{2}\right|^{2}\right\rangle \\
P_{C 3} & =\left\langle\left|\underline{k}^{T} \cdot \underline{e}_{3}\right|^{2}\right\rangle .
\end{aligned}
$$

Consequently, (21) can be modified as

$$
\left|\gamma_{d}\left(\underline{\omega}_{T}, \underline{\omega}_{P}\right)\right|=\frac{1}{\sqrt{1+\frac{|b|^{2}}{|a|^{2}} \frac{P_{C 2}}{P_{T}}+\frac{|c|^{2}}{|a|^{2}} \frac{P_{C 3}}{P_{T}}}} .
$$

The change of basis that makes $\underline{\omega}_{T}=[1,0,0]^{T}$ can be found by solving a system of equations.

An easier way to obtain (28), starting from any set of basis, considers the Gram-Schmidt orthonormalization [27], which sets $\underline{\omega}_{T}$ as one axis of a new basis set for the target space $S U(3)$. The new basis will consists of three unitary vectors $\underline{u}_{1}=\underline{\omega}_{T}, \underline{u}_{2}=\underline{\omega}_{C 2}$, and $\underline{u}_{3}=\underline{\omega}_{C 3}$, where $\underline{\omega}_{C 2}$ and $\underline{\omega}_{C 3}$ are two orthogonal components to $\underline{\omega}_{T}$ that are necessary to build up the three diagonal elements of the coherency matrix. Hence, $P_{T}, P_{C 1}$, and $P_{C 2}$ are calculated as

$$
\begin{aligned}
P_{T} & =\left\langle\left|\underline{k}^{T} \cdot \underline{u}_{1}\right|^{2}\right\rangle \\
P_{C 2} & =\left\langle\left|\underline{k}^{T} \cdot \underline{u}_{2}\right|^{2}\right\rangle \\
P_{C 3} & =\left\langle\left|\underline{k}^{T} \cdot \underline{u}_{3}\right|^{2}\right\rangle .
\end{aligned}
$$

With this operation, we complete the process that makes the detector a mathematical operator, where the optimum RedR's are set on the base of the $S C R$ and bias removal, as explained in the previous sections.

\section{Specialization to Multiple Reflection and Oriented Dipole}

The mathematical formulation shows that the algorithm is able to detect any single target as long as its PF (in particular the two Co-Pol Nulls) or Huynen parameters are known. To test the algorithm over real targets, the detection is specialized for multiple reflections (odd and even bounce) and oriented dipoles (horizontally and vertically). These four typologies of target are selected because of the relatively easy association with real targets on a radar image.

Fig. 6 represents the Poincaré sphere with characteristic polarizations for the targets considered.

\section{VALIDATION}

To validate and test the potential of the detector, it is applied on a fully polarimetric L-band SAR data set. In all the mathematical formulation, the frequency is not involved, and the detector is not directly frequency dependent (the dependence is related with changes in the target when the frequency is varied). The choice of the frequency can be related to the target to be detected. L-band presents an interesting setting based on its ability to penetrate foliage (FOLPEN capability) [28]. The data set was acquired by the German Aerospace Agency (DLR) 


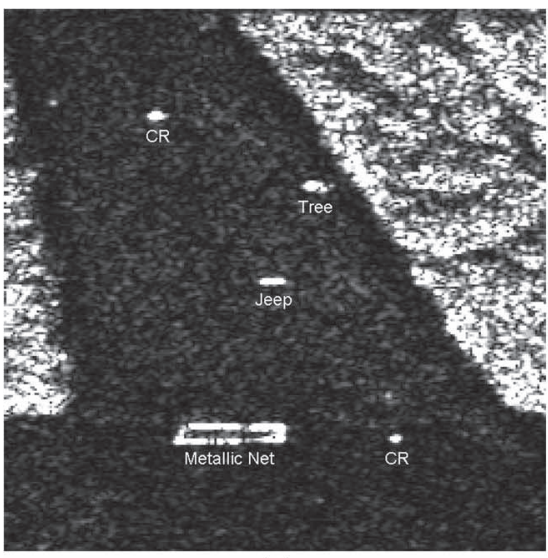

(a)

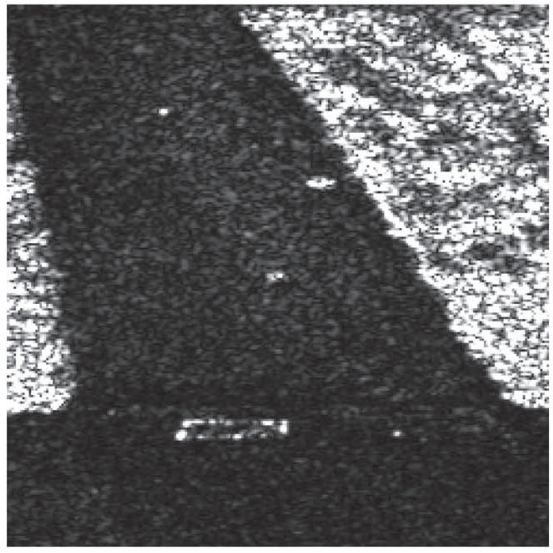

(d)

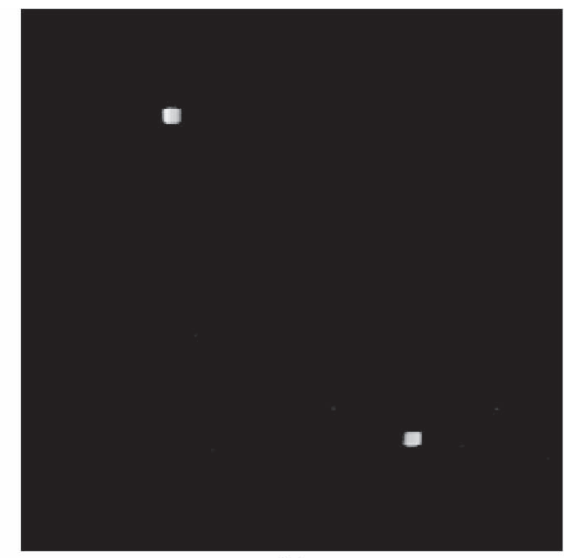

(b)

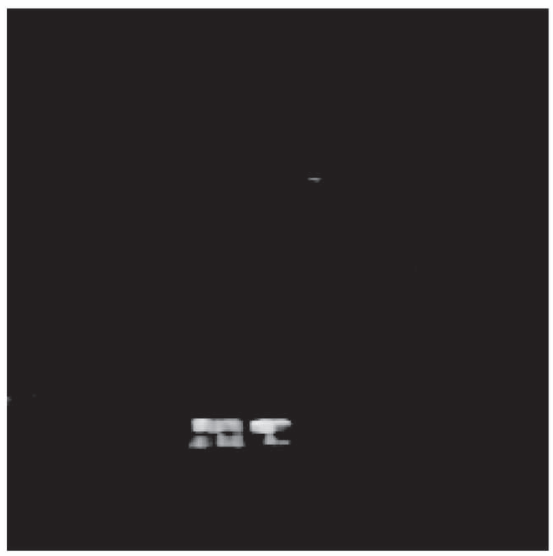

(e)

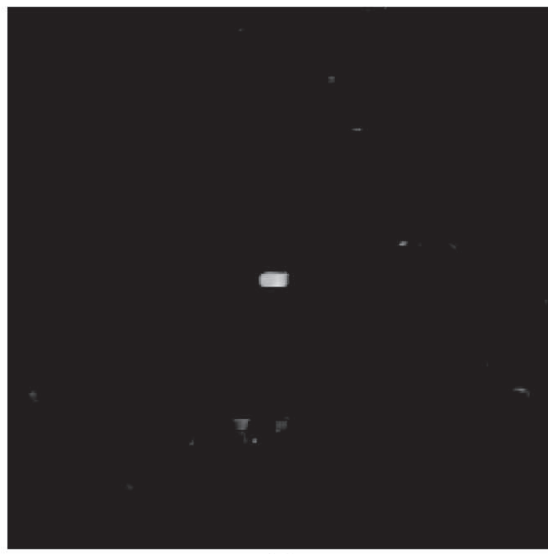

(c)

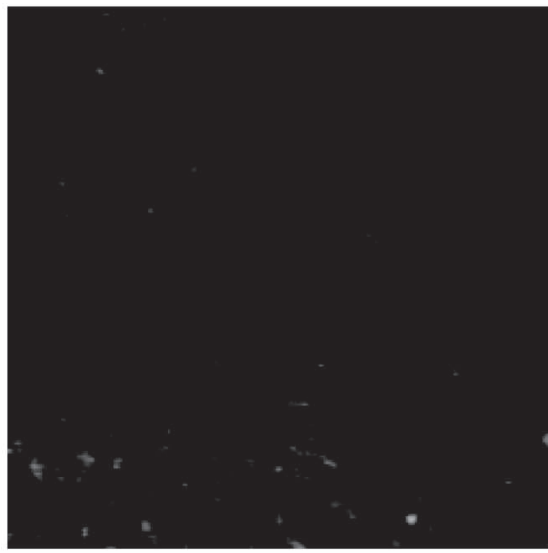

(f)

Fig. 7. Detection over an open field area. (a) L-band HH polarization with markers for some targets. (b) Mask for odd bounce detection (5 $\times 5$ ). (c) Mask for even bounce detection. (d) L-band HV polarization. (e) Mask for horizontal dipole detection. (f) Mask for vertical dipole detection. The intensity of the masks is related to the detector amplitude.

during the SARTOM campaign in 2006 [29] with the E-SAR airborne system.

One aim of the campaign was target detection beneath foliage; hence, a set of artificial targets were deployed in open fields and inside the forest. For this reason, the data set presents an ideal test scenario. As explained before, the threshold used is higher for open fields than for forested areas. Fig. 7 presents the detection on an open field. The L-band reflectivities in (a) $\mathrm{HH}$ and (d) $\mathrm{HV}$ polarizations are given for comparison. Moreover, in (a), there are markers to identify particular targets. A jeep is deployed in the middle of the image (Mercedes Benz 250 GD, also named "Wolf"), and the two bright points above and below the jeep are trihedral corner reflectors used for calibration (top, $149 \mathrm{~cm}$; bottom, $70 \mathrm{~cm}$ ). Finally, on the bottom of the image, there is a vertical metallic net (these defenses were used to delimitate areas). The range direction is along the vertical axes (bottom to top). The detector masks show where the targets are located, where the intensity is related to the amplitude of the detector (modified coherence amplitude), scaled to the base of the threshold. The detector parameters are those proposed in the previous section. The algorithm detects the trihedral corner reflectors as a source of odd bounce (b). The jeep mainly presents even bounces (presumably with the ground) (c). Moreover, we can see some even bounces on the forest edge due to the trunk-ground double-bounce effect that is stronger at the edge where it is exposed and has less attenuation from the canopy.

Finally, the net has a strange polarimetric behavior. Due to the mesh size (which goes from around $10 \mathrm{~cm}$ on the bottom to $30 \mathrm{~cm}$ on the top), the lower part is roughly similar to a wall for the $23-\mathrm{cm}$ wavelength radiation. It creates weak double bounce with the ground and strong horizontal dipoles (e). In fact, due to the radar geometry, the return from the net vertical dipole is much lower (f). Regarding the oriented dipole detection [(e) and (f)], the corner reflectors completely disappear (they are surfaces). Moreover, the horizontal branches of the isolated tree are visible (i.e., big horizontal branches throughout the canopy), as well as some vertical structure on the ground (i.e., bushes with big wooden vertical stems). A ground campaign was performed to check the existence of the targets detected-in the interests of brevity, we do not show the photographs of the targets.

In Fig. 8, the algorithm is tested for detection beneath foliage (FOLPEN). The targets are three trihedral corner reflectors (top: $149 \mathrm{~cm}$, bottom left: $70 \mathrm{~cm}$, and bottom right: $90 \mathrm{~cm}$ ). In the reflectivity images [(a) and (d)], the corner reflectors (CRs) are not recognizable; conversely, they are easily detected in (b) the odd bounce mask (i.e., triple bounces).

Considering that the threshold now is low, some points on the bare soil are detected as sources of single bounce [top of (b)]. These are not false alarms since the bare soil can be 


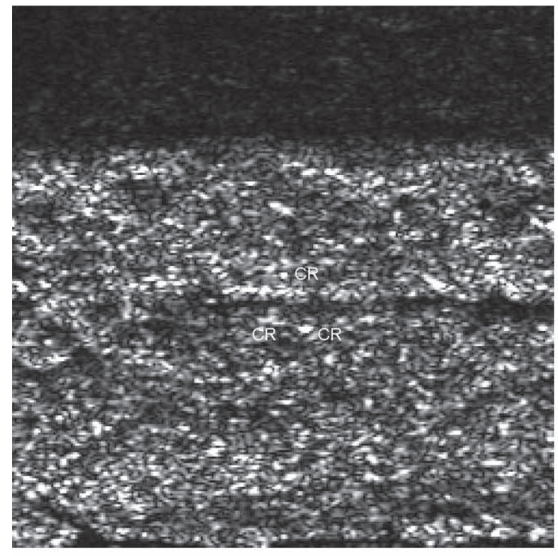

(a)

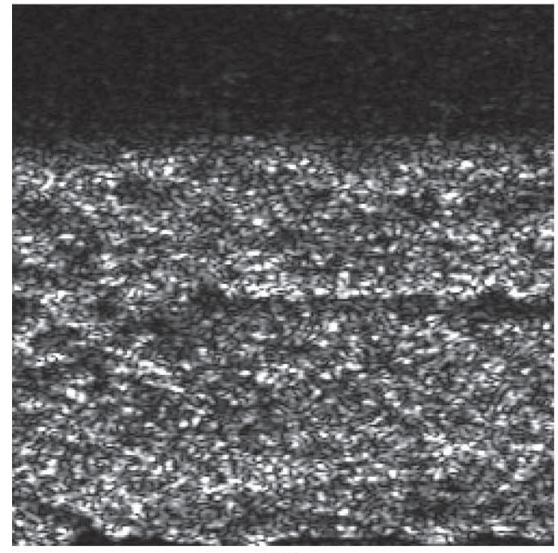

(d)

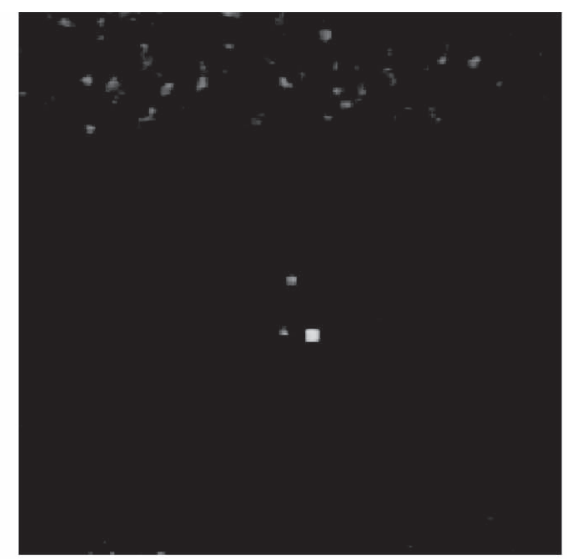

(b)

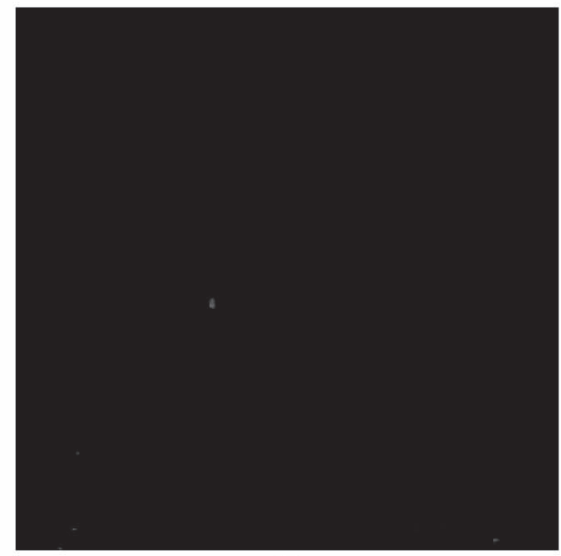

(e)

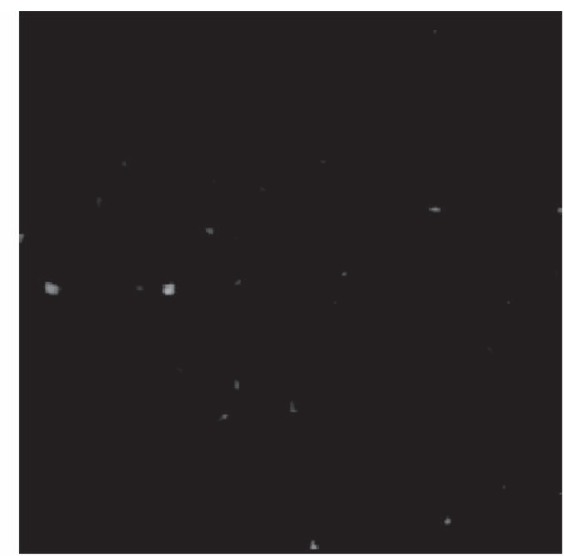

(c)

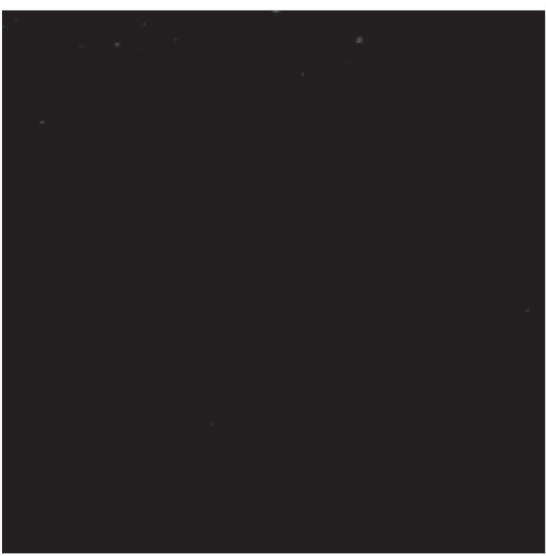

(f)

Fig. 8. Detection over forested area (same as Fig. 7). (a) L-band HH polarization. (b) Odd bounce. (c) Even bounce. (d) L-band HV polarization. (e) Horizontal dipoles. (f) Vertical dipoles.

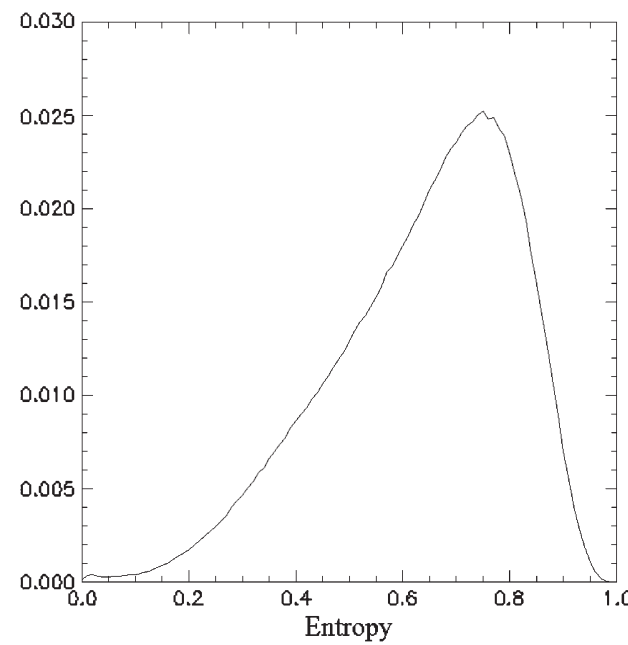

(a)

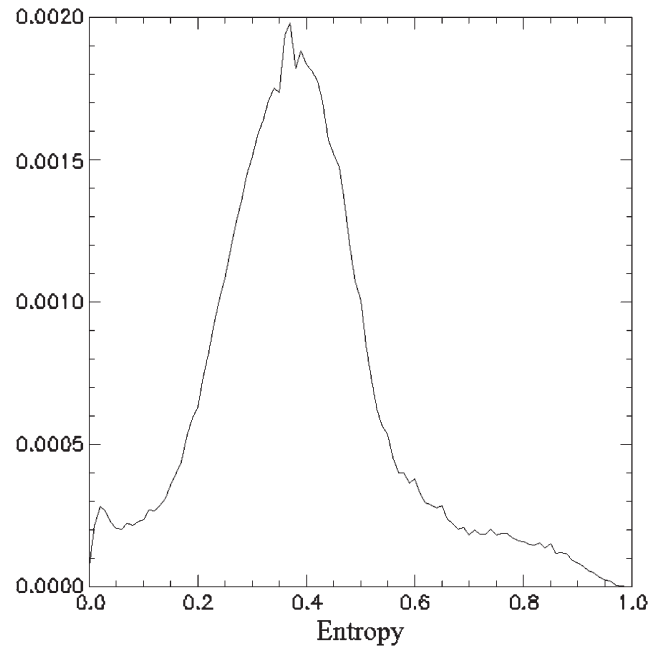

(b)

Fig. 9. Histogram of the entropy for (a) total image and (b) detected mask.

approximated as a single-bounce Bragg surface. Regarding the even bounce (c), it detects some trunk-ground double bounce, particularly in proximity of the forest clearing (which runs horizontally, separating the top and bottom CRs). Finally, it is not possible to detect particular oriented dipoles in the forest [(e) and (f)]. This is in line with the random volume over ground (RVoG) model for L-band, where the forest structures are random and do not present preferential orientations.

\section{A. Polarimetric Characterization of Detected Targets}

The algorithm development is based on the PF (or Huynen parameters). This means that the detectors are mainly aimed at single targets. In fact, an ideal PF cannot be defined for a partial target [24], [30]. To check this property, the entropy for the detected points over the whole data set is estimated, and the normalized histogram is presented in Fig. 9(b). The entropy is generally lower than 0.5 , indicating targets with single-scatterer 


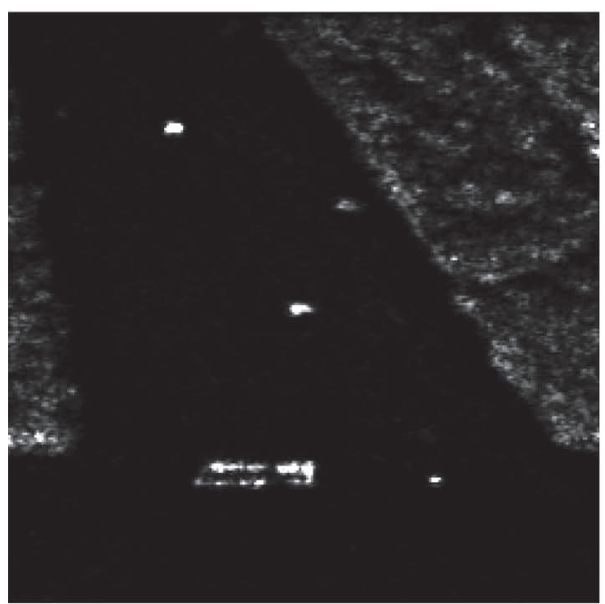

(a)

Fig. 10. PWF. (a) Open field. (b) Forested area.

(coherent) behavior. As a comparison, in Fig. 9(a), the entropy for all the pixels is depicted, showing much higher values.

\section{B. Comparison With PWF}

The aim of this section is to have a comparison with one of the most commonly used polarimetric target detector, i.e., polarimetric whitening filter (PWF) [4]. Briefly, the PWF uses the polarization to filter the images, reducing (optimally) the speckle. Practically, all the pixels interpreted as affected by speckle are strongly reduced. PWF is nowadays considered as one of the most powerful detectors that do not require a priori information about the statistics of the target. Since our detector does not require statistical a priori hypothesis as well, the comparison is worthwhile.

Fig. 10(a) and (b) shows the results of the PWF for the two areas already presented. In the open field, the performances are comparable (a). Both of the techniques detect jeep, net, and corner reflectors. However, PWF only performs target detection and not target classification. On the other hand, in a more critical situation as in a forested scenario, the PWF fails in detecting one CR (bottom left: $70 \mathrm{~cm}$ ). This is due to the fact that the embedded targets can be affected by speckle even if they are coherent themselves (because of the surrounding clutter and nonuniform attenuation). Regarding the weak targets, PWF is based on a threshold over the backscattered power; hence, weak targets are lost. The new detector proposed here is based on the weight of the target components; hence, it can detect low backscattering targets as long as they are polarimetrically characterized.

\section{CONCLUSion}

A target detector has been developed based on the unique polarimetric fork (PF) of the single target (similarly, the Huynen parameters or the $\alpha$ angle can be used). The mathematical formulation carried out is general and so can be applied for any single target of interest (as long as the PF is known). The validation was achieved over two categories of targets: multiple reflection and oriented dipoles. In both cases, the results are in line with the expected physical behavior of the targets. A

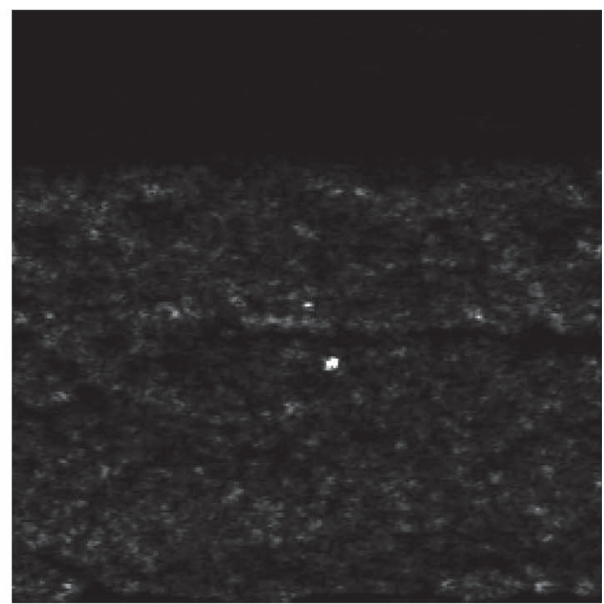

(b)

supplementary theoretical validation and evaluation is carried out wherein the algorithm is compared with the well-known PWF, showing better performances for embedded targets.

This paper presents the first attempt to use polarimetric filters to make a sensitivity analysis aimed at target detection. Regarding the application of the detector, the targets that can be investigated are not exclusively artificial. For instance, if the polarimetric model of a particular single target is available (we could eventually obtain it from a data set), the algorithm can be used to recognize similar features that appear elsewhere in another data set.

\section{ACKNOWLEDGMENT}

This paper forms part of the SARTOM project run by eOsphere Ltd and funded by DSTL. The authors would like to thank N. Walker of eOsphere for moral and technical support of the algorithm development and M. Brolly from the School of Geosciences, University of Edinburgh, for his help in the preparation of this paper.

\section{REFERENCES}

[1] L. M. Novak, G. J. Owirka, and A. L. Weaver, "Automatic target recognition using enhanced resolution SAR data," IEEE Trans. Aerosp. Electron. Syst., vol. 35, no. 1, pp. 157-175, Jan. 1999.

[2] J. Li and E. G. Zelnio, "Target detection with synthetic aperture radar," IEEE Trans. Aerosp. Electron. Syst., vol. 32, no. 2, pp. 613-627, Apr. 1996.

[3] L. M. Novak, G. J. Owirka, and C. M. Netishen, "Performance of a highresolution polarimetric SAR automatic target recognition system," Linc. Lab. J., vol. 6, pp. 11-24, 1993.

[4] L. M. Novak, M. C. Burl, and M. W. Irving, "Optimal polarimetric processing for enhanced target detection," IEEE Trans. Aerosp. Electron. Syst., vol. 29, no. 1, pp. 234-244, Jan. 1993.

[5] S. R. Cloude, D. G. Corr, and M. L. Williams, "Target detection beneath foliage using polarimetric synthetic aperture radar interferometry," Waves Random Complex Media, vol. 14, no. 2, pp. 393-414, Apr. 2004.

[6] G. D. De Grandi, J.-S. Lee, and D. L. Schuler, "Target detection and texture segmentation in polarimetric SAR images using a wavelet frame: Theoretical aspects," IEEE Trans. Geosci. Remote Sens., vol. 45, no. 11, pp. 3437-3453, Nov. 2007

[7] G. Margarit, J. J. Mallorqui, and X. Fabregas, "Single-pass polarimetric SAR interferometry for vessel classification," IEEE Trans. Geosci. Remote Sens., vol. 45, no. 11, pp. 3494-3502, Nov. 2007.

[8] W. M. Boerner, "Basics of radar polarimetry," RTO SET Lecture Series, 2004 
[9] I. H. Woodhouse, Introduction to Microwave Remote Sensing. Boca Raton, FL: CRC Press, 2006.

[10] S. R. Cloude and E. Pottier, "A review of target decomposition theorems in radar polarimetry," IEEE Trans. Geosci. Remote Sens., vol. 34, no. 2, pp. 498-518, Mar. 1996.

[11] E. M. Kennaugh and R. W. Sloan, "Effects of type of polarization on echo characteristics," Ohio State Univ. Res. Foundation Columbus Antenna Lab., Columbus, OH, B745559, Mar. 17, 1952.

[12] S. R. Cloude, "Polarimetry: The characterisation of polarisation effects in EM scattering," Ph.D. dissertation, Electron. Eng. Dept., Univ. York, York, U.K., 1987.

[13] J. R. Huynen, "Phenomenological theory of radar targets," Ph.D. dissertation, Tech. Univ. The Netherlands, Delft, The Netherlands, 1970.

[14] W. L. Cameron and L. K. Leung, "Feature motivated polarization scattering matrix decomposition," in Proc. Rec. IEEE Int. Radar Conf., 1990, pp. 549-557.

[15] E. Krogager, "Aspects of polarimetric radar imaging," Ph.D. dissertation, Tech. Univ. Denmark, Lyngby, Denmark, 1993.

[16] W. M. Boerner, M. B. El-Arini, C. Y. Chan, and P. M. Mastoris, "Polarization dependence in electromagnetic inverse problems," IEEE Trans. Antennas Propag., vol. AP-29, no. 2, pp. 262-271, Mar. 1981.

[17] W. M. Boerner, W. L. Yan, A. Q. Xi, and Y. Yamaguchi, "The characteristic polarization states for the coherent and partially polarized case," in Proc. IEEE ICAP, 1991, vol. 79, pp. 1538-1550.

[18] K. P. Papathanassiou, "Polarimetric SAR interferometry," Ph.D. dissertation, Tech. Univ. Graz, Graz, Austria, 1999.

[19] K. P. Papathanassiou and S. R. Cloude, "Single-baseline polarimetric SAR interferometry," IEEE Trans. Geosci. Remote Sens., vol. 39, no. 11, pp. 2352-2363, Nov. 2001.

[20] S. R. Cloude, "Lie groups in electromagnetic wave propagation and scattering," in Electromagnetic Symmetry, C. E. Baum and H. Kritikos, Eds. New York: Taylor \& Francis, 1995, pp. 91-142.

[21] S. R. Cloude and E. Pottier, "An entropy based classification scheme for land applications of polarimetric SAR," IEEE Trans. Geosci. Remote Sens., vol. 35, no. 1, pp. 68-78, Jan. 1997.

[22] G. A. Deschamps and P. Edward, "Poincare sphere representation of partially polarized fields," IEEE Trans. Antennas Propag., vol. AP-21, no. 4, pp. 474-478, Jul. 1973.

[23] Y. Dong and B. Forster, "Understanding of partial polarization in polarimetric SAR data," Int. J. Remote Sens., vol. 17, no. 12, pp. 2467-2475, Aug. 1996.

[24] J. van Zyl, C. Papas, and C. Elachi, "On the optimum polarizations of incoherently reflected wave," IEEE Trans. Antennas Propag., vol. AP-35, no. 7, pp. 818-825, Jul. 1987.

[25] R. Touzi, A. Lopes, J. Bruniquel, and P. W. Vachon, "Coherence estimation for SAR imagery," IEEE Trans. Geosci. Remote Sens., vol. 37, no. 1, pp. 135-149, Jan. 1999.

[26] G. Franceschetti and R. Lanari, Synthetic Aperture Radar Processing. Boca Raton, FL: CRC Press, 1999.

[27] G. Strang, Linear Algebra and Its Applications, 3rd ed. Stamford, CT: Thomson Learning, 1998

[28] J. G. Fleischman, S. Ayasli, and E. M. Adams, "Foliage attenuation and backscatter analysis of SAR imagery," IEEE Trans. Aerosp. Electron. Syst., vol. 32, no. 1, pp. 135-144, Jan. 1996.

[29] R. Horn, M. Nannini, and M. Keller, "SARTOM airborne campaign 2006: Data acquisition report," DLR, Braunschweig, Germany, Rep. DLR-HRSARTOM-TR-001, Dec. 2006.

[30] S. R. Cloude, "Uniqueness of target decomposition theorems in radar polarimetry," in Proc. Direct Inverse Methods Radar Polarimetry, 1992, pp. 267-296.

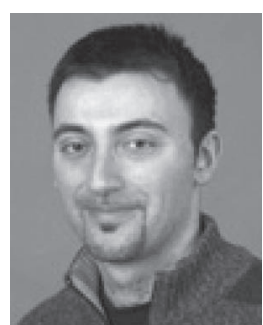

Armando Marino received the M.Sc. degree in telecommunication engineering from the Universita di Napoli "Federico II," Naples, Italy, in 2006. He is currently working toward the Ph.D. degree in the field of polarimetric SAR interferomery with the School of Geosciences, University of Edinburgh, Edinburgh, U.K.

In 2006, he was with the High Frequency and Radar Systems Department (HR), German Aerospace Center (DLR), Oberpfaffenhofen, Germany, where he developed his M.Sc. thesis, which focused on SAR multipass retrieval of forest parameters. His main research interest is target detection and POLInSAR applied on forested areas.

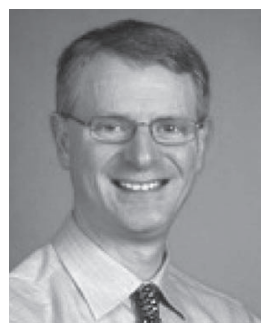

Shane R. Cloude (M'87-SM'96-F'01) received the B.Sc. degree from the University of Dundee, Dundee, U.K., in 1981 and the Ph.D. degree from the University of Birmingham, Birmingham, U.K., in 1987.

He was a Radar Scientist with the Royal Signals and Radar Establishment (RSRE), Great Malvern, U.K., until 1987. Following this, he held teaching and research posts with the University of Dundee, the University of York, York, U.K., and the University of Nantes, Nantes, France, before taking on his present role in 1996. He is currently the Director and a Senior Scientist with the Applied Electromagnetic Consultants (AELc), Edinburgh, U.K., undertaking contract research on a range of problems associated with radar remote sensing.

Dr. Cloude is a Fellow of the Alexander von Humboldt Society in Germany and an Honorary Fellow of the School of Geosciences, University of Edinburgh.

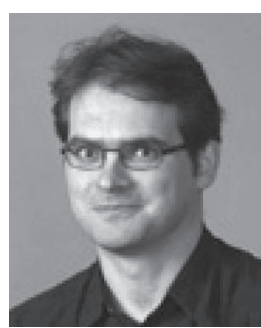

Iain H. Woodhouse (M'96) received the B.Sc. degree (Hons.) from the University of Edinburgh, Edinburgh, U.K., the M.Sc. degree in remote sensing from Dundee University, Dundee, U.K., and the Ph.D. degree from the Heriot-Watt University, Edinburgh.

He has worked with the Marconi Research Center and Wageningen University, Wageningen, The Netherlands. In 1999, he moved to the University of Edinburgh, where he is currently a Senior Lecturer in radar remote sensing with the School of GeoSciences. He is a Principal Investigator on a number of projects related to quantitative measurements of forests from remote sensing, which are funded by NERC, DTI, DSTL, and the Forestry Commission. His expertise is in the retrieval of biophysical properties of vegetation using active remote sensing, specifically synthetic aperture radar (SAR) and lidar. A key focus of his approach is the linking of observational models with process models.

Dr. Woodhouse is also a member of the NERC Earth Observation Director's Advisory Board and the Group on Earth Observations (GEO) Capacity Building Committee and a Nonexecutive Director of Ecometrica Ltd. He was a founding member of the Edinburgh Earth Observatory (EEO), a research group within the School of GeoSciences. 